\title{
The assistance of the iron porphyrin ligands to the binding interaction between the Fe center and small molecules in solution
}

\author{
Jie Xiao, ${ }^{, \dagger}$ Ronny Golnak, ${ }^{\dagger, \S}$ Kaan Atak, ${ }^{\dagger, \ddagger}$ Mika Pflüger, ${ }^{\dagger, \ddagger}$ Marvin Pohl, ${ }^{\dagger, \ddagger}$ Edlira Suljoti, \\ Bernd Winter, ${ }^{\dagger}$ Emad F. Aziz ${ }^{*,+,}$ \\ †oint Ultrafast Dynamics Lab in Solutions and at Interfaces (JULiq), Helmholtz-Zentrum Berlin für \\ Materialien und Energie, Albert-Einstein-Strasse 15, D-12489 Berlin, Germany \\ ${ }^{\ddagger}$ Freie Universität Berlin, Fachbereich Physik, Arnimallee 14, D-14195 Berlin, Germany \\ ${ }^{\S}$ Freie Universität Berlin, Fachbereich Biologie, Chemie, Pharmazie, Takustrasse 3, D-14195 Berlin, \\ Germany
}

Keywords: iron porphyrin, ligand, electronic structure, liquid solution, RIXS, XAS, XES, DFT/ROCIS

\begin{abstract}
Solute-solvent electronic structure interactions of iron-porphyrin at very low concentration in dichloromethane $\left(\mathrm{CH}_{2} \mathrm{Cl}_{2}\right)$ liquid solution are reported. Two iron porphyrin complexes are investigated here, iron octaethylporphyrin chloride (FeOEP-Cl) and iron tetraphenylporphyrin chloride (FeTPP-Cl), using X-ray absorption and emission spectroscopy at the $\mathrm{Fe}_{2,3}$ edge, and spectra are interpreted with the help of density functional theory/restricted open shell configuration interaction singles (DFT/ROCIS) calculations. It is argued that the Fe center of FeOEP-Cl is more capable of binding small solvent molecules, exemplified here for $\mathrm{Cl}_{2} \mathrm{CH}_{2}$, than FeTPP-Cl in solution. The proposed binding mechanism is through the assistance of the dipole interaction between the porphyrin-ligand system and the solvent molecule, in a situation where the ligand structure and arrangement maximize the binding interactions. Our studies demonstrate that even small ligands, depending on their structure and arrangement, can have considerable effects on porphyrin's metal center chemistry in liquid solution.
\end{abstract}

\section{Introduction}

Hemoglobin and myoglobin play a vital role in biological systems, responsible for binding and transporting oxygen molecules, $\mathrm{O}_{2}$, in which iron porphyrin is the major functional part with its Fe center acting as the absorption site. ${ }^{1-5}$ In an oxygen-molecule binding process, $\mathrm{O}_{2}$ becomes superoxide ion, $\mathrm{O}_{2}{ }^{-}$, once it binds to the Fe site, while Fe is oxidized from $2+$ to $3^{+}$oxidation state simultaneously, according to Weiss model..$^{6-8}$ This process creates an ion-induced dipole force between $\mathrm{Fe}^{3+}$ and $\mathrm{O}_{2}^{-}$, also known as dipolar bond that keeps oxygen molecule closely attached during the transportation. Many investigations have been carried out aiming at better understanding the binding characteristic of Fe with $\mathrm{O}_{2}$, and also other small molecules, such as $\mathrm{Fe}-\mathrm{CO}, \mathrm{NO}, \mathrm{SO}, \mathrm{CN}$ in hemoglobin (or myoglobin). ${ }^{7}{ }^{12}$ The influence of the protein surrounding iron porphyrin in hemoglobin or myoglobin on the bonding nature of Fe with $\mathrm{O}_{2}$ and $\mathrm{CO}$ has also been investigated. ${ }^{5,8}$ Multiple effects from the protein have been identified, including steric hindrance, hydrogen bonding, and local polarity. ${ }^{1,13-17}$ However, due to the large size and the complex structure of protein, the interaction of protein with small molecules becomes extremely complicated. In addition, little attention has been paid to the small side ligands of porphyrin molecule itself. It is still unclear if there are contributions from the porphyrin side ligands to the small molecule binding process. It is of fundamental importance, to gain a clear and 
comprehensive understanding of the structural and dynamic properties of this binding interaction, both experimentally and theoretically, under realistic conditions.

In order to specifically explore possible ligand effect in the present study, several experimental requirements need to be fulfilled. First, both porphyrin and small molecule need to be embedded in liquid phase to mimic their mutual interactions under realistic conditions where molecules can be highly mobile. ${ }^{12}$ Second, iron porphyrin with simple, well-defined, and symmetric ligands should be considered in order to avoid additional complexity in the ligand interactions with small molecules, as would be the case of heme in hemoglobin or myoglobin. Third, to further detail the interaction between the Fe center and small molecules, it is advantageous if only one type of small molecules is present in the solution to rule out possible cooperative or competitive binding to the Fe site., ${ }^{5,18}$ Given these requirements, two iron porphyrin complexes, iron octaethylporphyrin chloride (FeOEP-Cl) and iron tetraphenylporphyrin chloride (FeTPP-Cl), both dissolved in dichloromethane $\left(\mathrm{CH}_{2} \mathrm{Cl}_{2}\right)$ solvent, were selected. The molecular structures of porphyrins are shown in figure 1. There are three main advantages of our choice: 1) The Fe center in porphyrin is already at $3^{+}$ oxidation state. It could readily bind one of the $\mathrm{Cl}$ (since $\mathrm{Cl}$ is negatively charged, although its partial charge is smaller than that of the $\mathrm{Cl}^{-}$in original porphyrin) from a solvent molecule without much redox reaction, thus forming a $\mathrm{Fe}^{3+}-\mathrm{Cl}^{-}$pair analogue to $\mathrm{Fe}^{3+}-\mathrm{O}_{2}{ }^{-}$pair. 2) Only one type of the side ligands exists in each porphyrin molecule, eight ethyl groups in FeOEP-Cl and four phenyl groups in FeTPP-Cl. This rules out additional interactions that may occur between dissimilar ligands. 3) Only one type of small molecules, dichloromethane $\mathrm{CH}_{2} \mathrm{Cl}_{2}$, is present in both sample solutions, which interacts with the $\mathrm{Fe}$ center, and serves as solvent at the same time. Our choice of $\mathrm{CH}_{2} \mathrm{Cl}_{2}$ as solvent is also driven by the considerably higher solubility of this chlorinated molecule as compared to other commonly used organic solvents. This is important here in order to obtain reasonably large solute signal in the X-ray absorption spectra. Furthermore, we have observed that the chemical interactions of $\mathrm{CH}_{2} \mathrm{Cl}_{2}$ with the $\mathrm{Si}_{3} \mathrm{~N}_{4}$ membrane of the liquid flow-cell are much reduced than in the case of aqueous solutions. As a consequence, for the present solution, degradation and/or breaking of the membrane does not occur upon intense synchrotron radiation during the measurements. If a small solvent molecule binds to the Fe site, the Fe center will be coordinated by six atoms (four $\mathrm{N}$ and two $\mathrm{Cl}$ ) forming a distorted octahedron. According to $\mathrm{O}_{h}$ symmetry, the resulting ligand field leads to a splitting of the Fe $3 \mathrm{~d}$ orbitals into two groups: $e_{g}\left(\mathrm{~d}_{\mathrm{x} 2-\mathrm{y} 2}, \mathrm{~d}_{\mathrm{zz}}\right)$ and $t_{2 g}\left(\mathrm{~d}_{\mathrm{xz}}, \mathrm{d}_{\mathrm{yz}}, \mathrm{d}_{\mathrm{xy}}\right)$, corresponding to the two major absorption peaks observed in our X-ray absorption measurements, as shown in figure $2 \mathrm{~A}$ (discussed below). ${ }^{9,10,19-21}$

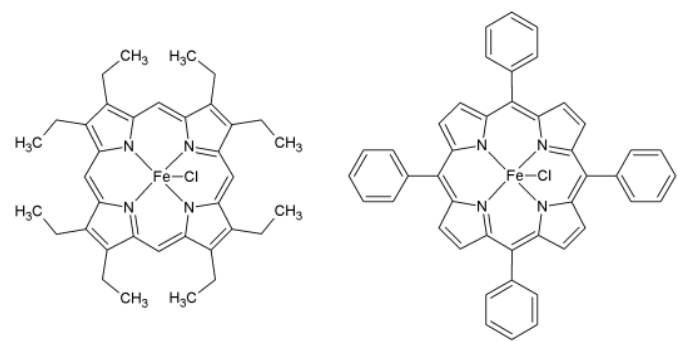

Figure 1. Structural formula of FeOEP-Cl (left) and FeTPP-Cl (right).

Since binding occurs solely at the Fe site, a site (element) specific probing technique is desirable, making X-ray spectroscopy the most suitable probing tool for liquid samples. $^{22-24}$ X-ray absorption spectroscopy (XAS) and resonant inelastic X-ray scattering (RIXS), with the excitation photon energy tuned to the Fe $\mathrm{L}_{2,3}$ edge, were employed here to explore the iron $3 \mathrm{~d}$ electronic structure of the two porphyrin molecules, in which unoccupied valence levels and $3 \mathrm{~d}$ excitations were measured respectively. ${ }^{25}$ The photon-in (excitation) photon-out (probing) process makes our measurements bulk sensitive, significantly reducing possible interface contributions. Compared with the K-edge measurements on transition metal complexes, the Fe $2 \mathrm{p}$ to $3 \mathrm{~d}$ transition studied here is dipole allowed, yielding more intense and more structured spectra associated with $3 \mathrm{~d}$ orbitals than for the dipole forbidden K-edge transitions. ${ }^{25}$ Since there only exist two types of molecules in each sample solution, any significant differences, if observed, in the Fe L-edge XAS and/or RIXS spectra of these two solutions must originate from the side ligands. 
Differences are indeed detected, and the specific role played by the ligands is corroborated by our density functional theory/restricted open-shell configuration interaction singles (DFT/ROCIS) calculations.

\section{Experiment}

Iron octaethylporphyrin chloride (FeOEP-Cl) and iron tetraphenylporphyrin chloride (FeTPP-Cl) powder samples with 98\% purity purchased from TriPorTech were dissolved in solvent dichloromethane $\left(\mathrm{CH}_{2} \mathrm{Cl}_{2}\right)$ with $>99.9 \%$ purity from Sigma Aldrich to prepare the solutions of $15 \mathrm{mM}$ and $25 \mathrm{mM}$ concentrations, respectively. The porphyrin solution was continuously circulated through a flow-cell during the measurements to avoid possible beam damage. A 100-nm thick $\mathrm{Si}_{3} \mathrm{~N}_{4}$ membrane was used as the flow cell window to allow X-ray to penetrate in and out. ${ }^{12}$

The experiment was carried out at the U41-PGM undulator beamline and the LiXEdrom endstation at the synchrotron facility BESSY II, Berlin. The U41-PGM provides horizontally linear polarized light with high photon flux $\left(\sim 10^{13}\right.$ photons/sec $)$ and small focal spot $(-40 \mu \mathrm{m}$ of vertical size $)$, which is crucial to our measurements. High flux is required for low-concentration samples, and also when using a flow-cell with its membrane absorbing some fraction of the transmitted photons. Small vertical focal size $\left(\begin{array}{ll}\sim & \mu \mathrm{m}\end{array}\right)$ assures high energy resolution for our photon detection. The photons emitted from the sample were collected along the polarization direction of the incident photon beam (to suppress the elastic peak) through a pinhole, and subsequently dispersed by a spherical grating with 1200 lines/mm and 7.5 $\mathrm{m}$ radius. Finally, the dispersed photons were detected by a multi-channel plate (MCP)/fluorescence screen/CCD assembly. ${ }^{26}$ The flow-cell was mounted with its membrane surface at $45^{\circ}$ with respect to the incoming and outgoing photon directions. The sample, grating, and photon detector are arranged on Rowland circle geometry for accurate focusing. The grating and detector chamber was kept at $10^{-8}$ mbar or lower to protect the grating surface and the MCP from contamination, while the sample chamber was at $10^{-7}$ mbar.

Partial fluorescence yield (PFY) was adopted for XAS measurement in order to reduce self absorption and saturation effects which is common for total fluorescence yield (TFY) detection. ${ }^{27,28}$ The incident photon energy was tuned to the $\mathrm{Fe} \mathrm{L}_{2,3}$ edge, while only the Fe $3 \mathrm{~d}->2 \mathrm{p}$ transition was selected for the detection of the emitted photon in both XAS (PFY) and RIXS measurements. The first diffraction order of the grating was chosen due to the low fluorescence yield signal. Since intense photon flux was focused to a tiny beam spot on the flow-cell membrane, thermal effects were inevitable in a long-time exposure of the same sample spot to X-ray. We found though that the spectra remained unchanged if the measurement from a given spot did not exceed $20 \mathrm{~min}$. All spectra presented here are the sum of the measurements obtained from different membrane positions with each less than 20 min exposure to X-ray.

\section{Computation}

The DFT calculations for exploring the electronic structure of the Fe site of iron porphyrin molecules were performed with the ORCA program package. ${ }^{29}$ Molecular geometry optimizations were carried out using B3LYP density functional method with def2-TZVP(-f) basis set. ${ }^{30^{-32}}$ The Fe $2 \mathrm{p}$ to valence level transitions were calculated with DFT/ROCIS using the same basis set and parameters $c_{1}=0.18, c_{2}=0.20$, and $c_{3}=0.40 .^{33,34}$ During the geometry-optimization calculations, the resolution of identity approximation was employed with the def2-TZV/J basis set. ${ }^{35}{ }^{40}$ Numerical integrations in the DFT calculations were performed on a dense grid (ORCA grid4). Fe L-edge absorption spectra, with spin-orbit coupling both included and excluded, were simulated by the summation of each DFT/ROCIS-calculated transition moment with a Gaussian type broadening of $1 \mathrm{eV}$. With spin-orbit coupling, thousands of transitions are created, making the simulated spectra almost impossible to interpret. The simulated L-edge spectra without spin-orbit coupling have much fewer transitions, without losing or distorting major spectroscopic features on $\mathrm{L}_{3}$ edge. Thus contributions from unoccupied states to the absorption transitions can be more easily identified. $^{33}$ Vibronic interference effects were not taken into account in the calculations.

\section{Results and discussion}


Obtained PFY and RIXS spectra of FeOEP-Cl and FeTPP-Cl are shown in figure 2, in blue and red, respectively. Although both molecules have the same nominal $\mathrm{Fe}^{3+}$ oxidation state, an overall energy shift of 0.4 $\mathrm{eV}$ to lower excitation energy of the FeOEP-Cl PFY spectrum is observed when compared with the FeTPP-Cl PFY spectrum, as marked by green dashed lines in figure $2 \mathrm{~A}$. Such a shift suggests that the actual Fe oxidation state in FeOEP-Cl is somehwat lower than that in FeTPP-Cl. Another spectroscopic feature in these two molecules' PFY spectra, although barely quantifiable due to poor signal-to-noise ratio, or its existence even being disputable without additional information, is the changed relative intensity ratio of the two leading absorption peaks, marked by the same green dashed lines mentioned above. We do argue thought that the differences of the peak-intensity ratio observed in the PFY spectra are indeed expected, and are in line with the RIXS measurements at the corresponding excitation energies $708.8 \mathrm{eV}$ and $710.5 \mathrm{eV}$, as we will demonstrate below. The small peak in the FeOEP-Cl PFY spectrum near $705.5 \mathrm{eV}$ is considered statistically insignificant. Quantification of further spectral differences in Figure $2 \mathrm{~A}$ for the two porphyrins, especially at the $\mathrm{L}_{2}$ edge, would be difficult, given the low signal-to-noise ratio. We remind that the low solute concentration requires a total data acquisition time of approximately 12-14 $h$ per PFY spectrum, as the ones presented in figure $2 \mathrm{~A}$.

The corresponding RIXS spectra, in figure $2 \mathrm{~B}$, exhibit broad featureless peaks in general which is constant at a certain emission energy for each absorption edge, indicating that the Fe 3d electronic systems in both porphyrins have little correlation. ${ }^{25}$ That is, the $\mathrm{Fe}$ 3d valence orbitals appear to be delocalized over the ligands for both molecules. Despite the rather similar RIXS spectra for the two different molecules at most excitation energies, RIXS spectra at $708.8 \mathrm{eV}$ and $710.5 \mathrm{eV}$ in figure $2 \mathrm{~B}$ unambiguously reveal distinctly different behavior in the relative intensity. We observe an opposite trend in signal intensities, i.e., larger overall intensity for the FeOEP-Cl than for FeTPP-Cl at $708.8 \mathrm{eV}$ excitation energy, and the reverse for $710.5 \mathrm{eV}$. Such an effect cannot be explained by the different concentrations only. Unlike the XA spectra of figure $2 \mathrm{~A}$, the RIXS spectra, measured here at few selected excitation energies and over an extended sampling time, exhibit good signal-to-noise ratio, and the quantitative comparison of the signal intensities for the two porphyrins is well justified. Note that signal integration of the RIXS spectrum at a given excitation energy yields the PFY spectrum; for the measurement of the actual PFY spectrum of figure $2 \mathrm{~A}$, each data point (integrated from an actual RIXS spectrum) had to be collected over much shorter time though which is the reason for the unsatisfactory signal level. Hence, above mentioned opposite intensity trends in the good-quality RIXS spectra for excitation at the two leading XA peaks, supports a corresponding intensity change in the PFY spectra; experimentally this effect cannot be unequivocally resolved here, as we have argued above. This different intensity ratio for the two leading absorption peaks together with the aforementioned $0.4 \mathrm{eV}$ energy shift in figure $2 \mathrm{~A}$ strongly indicates that the $\mathrm{Fe}$ ions in the two porphyrin centers have different chemical environments, and hence different ligand fields altering the electronic structure of the Fe site.

The geometry optimizations from our DFT calculations, however, give almost identical bond distances and bond angles of Fe-N and $\mathrm{Fe}-\mathrm{Cl}$ for both porphyrins (see scheme $\mathrm{S}_{1}$ in the Supporting Information), which should result in very similar electronic structure of the Fe centers for both molecules (see discussions for figure 3 , below). So the different ligand fields around the two porphyrin Fe centers are very unlikely caused by their original molecular geometric structures. Since the Fe center in each porphyrin molecule still has one absorption site vacant (opposite to the $\mathrm{Cl}^{-}$in the original porphyrin), we hypothesize that the Fe ion in one of the porphyrin molecular centers has a stronger interaction with solvent molecules through that site than in the other, resulting in different ligand fields due to the different Fe coordinates of the octahedron. Since it is the FeOEP-Cl PFY spectrum (blue trace in figure $2 \mathrm{~A}$ ) being shifted $0.4 \mathrm{eV}$ to lower excitation energy, $\mathrm{Cl}$ from $\mathrm{CH}_{2} \mathrm{Cl}_{2}$ is thus most likely to bind to the $\mathrm{Fe}$ center of $\mathrm{FeOEP}-\mathrm{Cl}$, and donates some electron charge to the $\mathrm{Fe}^{3+}$ ion. This slightly lowers the actual 
oxidation state of the $\mathrm{Fe}^{3+}$ in FeOEP-Cl. For FeTPP-Cl, on the other hand, such an interaction of $\mathrm{Fe}$ center with small solvent molecules seems to be negligible, or is at least less significant than in the FeOEP-Cl solution.

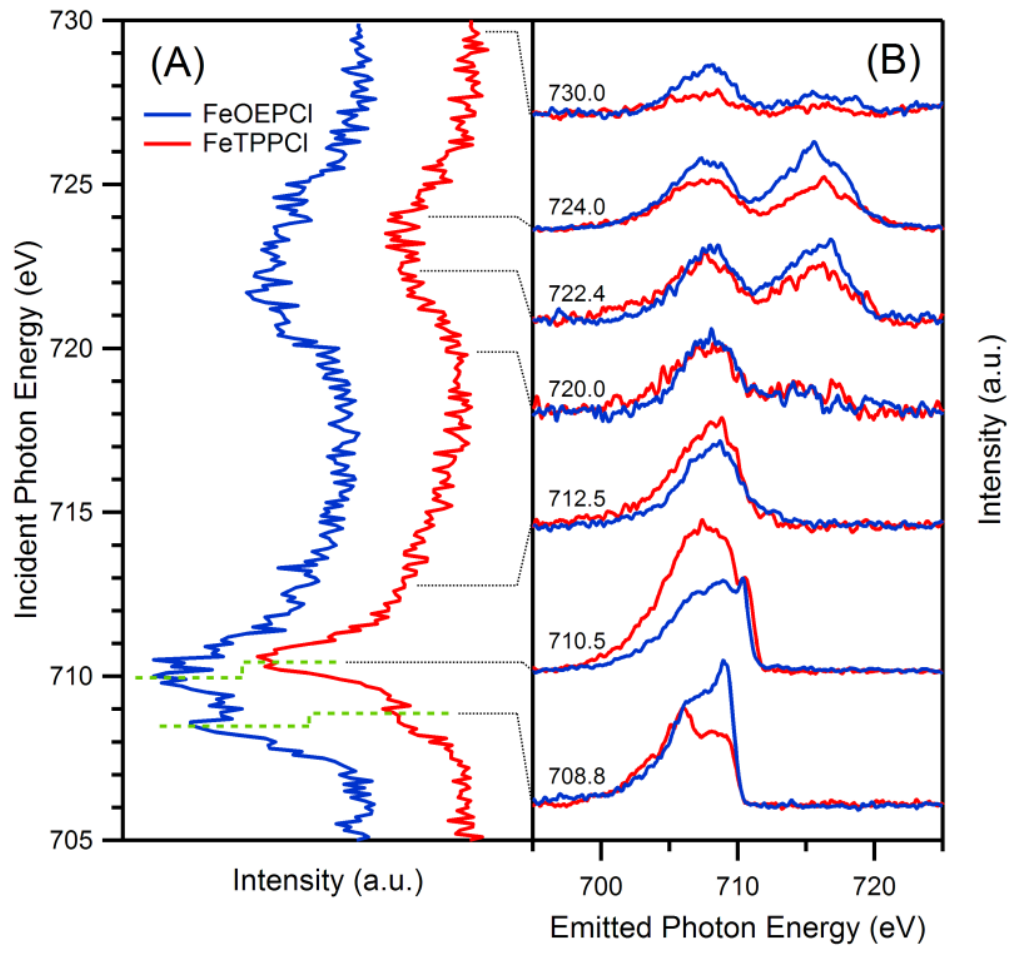

Figure 2. (A) Partial fluorescence yield (PFY) of FeOEP-Cl with $15 \mathrm{mM}$ concentration (blue trace) and FeTPP-Cl with $25 \mathrm{mM}$ concentration (red trace), both dissolved in dichloromethane $\left(\mathrm{CH}_{2} \mathrm{Cl}_{2}\right)$. Green dashed lines mark the first two major absorption peaks and indicate an overall $0.4 \mathrm{eV}$ shift relative to each other. (B) RIXS spectra of two porphyrins at indicated excitation energies. All RIXS spectra are plotted against emission photon energy instead of loss energy due to the constant emission energies of spectroscopic features at various excitation energies. Each RIXS spectrum is normalized to its background signal for intensity comparison with others.

The DFT/ROCIS calculations for various molecular configurations also support our above hypothesis that the two Fe centers have considerably different affinities of binding $\mathrm{Cl}$ from solvent molecule. The various molecular configurations include three possible $\mathrm{Fe}-\mathrm{Cl}$ bonding situations, each of which has three possible spin multiplicities. The original $\mathrm{Cl}^{-}$ion in both porphyrin complexes either remains bound to $\mathrm{Fe}^{3+}$, or the halide detaches when dissolved in the polar solvent dichloromethane. The $\mathrm{Cl}$ from solvent $\mathrm{CH}_{2} \mathrm{Cl}_{2}$ may also bind to $\mathrm{Fe}^{3+}$ to complete all six coordination sites around the $\mathrm{Fe}$ center in an approximate octahedral symmetry. Therefore, three $\mathrm{Fe}-\mathrm{Cl}$ bonding situations in solution can be distinguished: Fe binding to (1) no $\mathrm{Cl},(2)$ one $\mathrm{Cl}$, or (3) two $\mathrm{Cl}$ ions. Since $\mathrm{Fe}^{3+}$ oxidation state has $\mathrm{d}^{5}$ electronic configuration, there are three possible spin multiplicities for each $\mathrm{Fe}-\mathrm{Cl}$ bonding situation, i.e. $2 \mathrm{~s}+1=2,4,6 .^{4^{-}-44}$ Therefore, a total of nine different molecular configurations may exist for each porphyrin complex when dissolved in $\mathrm{CH}_{2} \mathrm{Cl}_{2}$ solvent. All nine structures were calculated, and are presented in figures $\mathrm{S}_{1}$ and $\mathrm{S}_{2}$ (see Supporting Information), along with the respective experimental PFY spectrum shown at the top of each figure for comparison. It is noted that the theoretical simulation of the two- $\mathrm{Cl}$ case (3) was done for the molecular structure of two isolated $\mathrm{Cl}^{-}$ions attached to the Fe center from opposite sides. This symmetry, corresponding to maximized interaction between the Fe center and the $\mathrm{Cl}^{-}$from the solvent molecule, is the preferred structure obtained from geometry optimization. Our 
simple structure model represents one single relevant snapshot of the many existing dynamical structures in the real solution, but it is yet useful in the theoretical simulation of the qualitative binding interactions. We believe that our simple model is unsuitable though to predict accurate bond strengths, or solute and ligand contributions to the bond. Quantification of these effects would exceed our current computational capabilities.

Overall shapes of the measured PFY spectra are yet distinct enough to allow for the quantitative comparison with the calculated spectra (see figures $\mathrm{S}_{1}$ and $\mathrm{S}_{2}$ in the Supporting Information). The best matching theoretical calculations, along with their experimental spectra, are reproduced in figure 3 for both porphyrins. Colors in figures $3 \mathrm{~b}$ and $3 \mathrm{e}$ follow the same color codes used in figures $\mathrm{S}_{1}$ and $\mathrm{S}_{2}$ in the Supporting Information. Judged by the peak positions and intensities of the first two leading absorption peaks at the $\mathrm{L}_{3}$ edge, marked by green dashed lines in figure 3 (and also in figures $\mathrm{S}_{1}$ and $\mathrm{S}_{2}$ ), the best matching calculated spectrum for FeTPP-Cl is the one from the original molecular structure (Fe binds to a single $\mathrm{Cl}$ ) with high spin multiplicity $2 \mathrm{~s}+1=6$, as shown in figure $3 \mathrm{e}$ (also the top blue trace in figure S2), and importantly no other calculated spectrum matches the experimental data, or would contribute significantly to it. For FeOEP-Cl, the same computed spectrum of the original molecular structure with high spin multiplicity 6 (blue trace in figure 3 b) seems to also reproduce the respective experimental data. However, the blue trace in figure $3 \mathrm{~b}$ (also the top blue trace in figure $\mathrm{S}_{1}$ ) is almost identical to figure $3 e$, and this cannot explain the observed difference of the intensity ratio of the two leading absorption peaks for two porphyrin complexes. Hence, another theoretical spectrum, pink trace in figure $3 \mathrm{~b}$ (same as the top pink trace in figure $\mathrm{S}_{1}$ ), representative of a configuration of $\mathrm{Fe}$ binding to two $\mathrm{Cl}$ with high spin multiplicity 6 , needs to be invoked to account for the relatively enhanced first absorption peak at $708.4 \mathrm{eV}$ for FeOEP-Cl. Moreover, the larger distance between the first and second absorption peak positions in the pink trace than found in the blue trace of figure $3 \mathrm{~b}$ could further explain the broader second absorption peak at $710.5 \mathrm{eV}$ of the FeOEP-Cl PFY spectrum when compared to the FeTPP-Cl PFY spectrum, as shown in figures 2A and 3a, 3d.

Traces in figures $3 c$ and $3 f$ are the calculations without spin-orbit coupling perturbation, which significantly reduces the number of transitions, and allows to identify the unoccupied states contributing most to the X-ray absorption transitions at the $\mathrm{Fe} \mathrm{L}_{2,3}$ edge. Five unoccupied $\mathrm{Fe} 3 \mathrm{~d}$ orbitals, indicated inside the green squares in figure 3 , are found to have the most significant contributions to the observed X-ray absorption. The first absorption peak located near $708.4 \mathrm{eV}$ for the FeOEP-Cl complex, and $708.8 \mathrm{eV}$ for the FeTPP-Cl complex, mostly originate from $t_{2 g}$ orbitals, while the second absorption peak at approximately $710.5 \mathrm{eV}$ for both porphyrins arises from $e_{g}$ orbitals, as indicated by green dashed squares in figure 3 . This is consistent with the assignment of our previous calculations using a different method. ${ }^{10}$ We refer to table $\mathrm{S}_{1}$ as well as figure $\mathrm{S}_{3}$ in the Supporting Information for the detailed information about atomic and orbital contributions to each molecular orbital. Despite the quite good agreement between the theoretical simulations and the experimental spectra, binding strengths and the percentage of the two $\mathrm{Cl}$ bound complexes for FeOEP-Cl cannot be determined here, using a very simple structure model as explained above. In addition to the different binding situations, charge transfer at the Fe site also affects the relative peak intensities. ${ }^{21}$ It will reduce the relative intensity of $t_{2 g}$ spectral feature (the marked absorption peak around $708.8 \mathrm{eV}$ ), as is indeed observed when comparing figure $3 \mathrm{~d}$ with figure ze for FeTPP-Cl. We are not able to quantify charge transfer on the level of the theory performed here. Given the almost identical geometrical structures for the central part of the two porphyrins (scheme S1 in Supporting Information), charge transfer is argued to lead to noticeable spectral differences only due to the extra binding discussed here. 

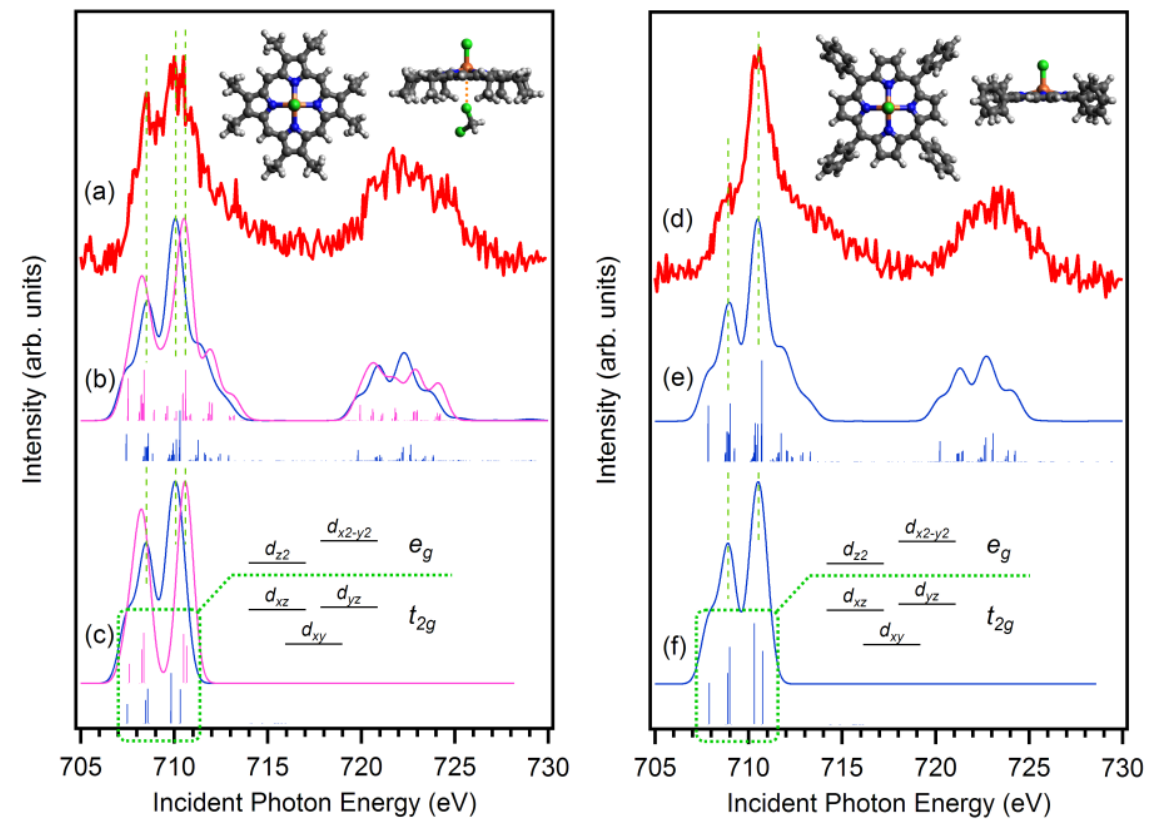

Figure 3. PFY spectra of FeOEP-Cl (a) and FeTPP-Cl (d), identical to the blue trace and red trace in figure $2 \mathrm{~A}$ respectively, compared with their corresponding DFT/ROCIS calculations in the left and right panels for each porphyrin molecule. The calculated spectra (b) and (e) are duplicates of the top pink and top blue traces in figure $\mathrm{S}_{1}$ for $\mathrm{FeOEP}-\mathrm{Cl}$ and top blue trace for $\mathrm{FeTPP}-\mathrm{Cl}$ in figure $\mathrm{S}_{2}$ with spin-orbit coupling perturbation included. (c) and (f) are calculated XAS spectra at $\mathrm{L}_{3}$ edge only without spin-orbit coupling for each complex. The vertical bars under each simulated spectrum represent the transition moments from Fe $2 \mathrm{p}$ to valence orbitals. The insets on the top of the figure are the top and side views of the molecular structures after geometry optimizations. For FeOEP-Cl, an extra binding interaction of the Fe center with a solvent molecule $\mathrm{CH}_{2} \mathrm{Cl}_{2}$ is also illustrated in the left inset.

The inclusion of an additional binding interaction between the $\mathrm{Fe}^{3+}$ center and a $\mathrm{Cl}$ from solvent molecule $\mathrm{CH}_{2} \mathrm{Cl}_{2}$ for FeOEP-Cl in solution, as quantified in our DFT/ROCIS calculations, well explains the observed differences of spectroscopic features in figure 2A. As mentioned above, any experimental and theoretical differences inferred from the comparison of the two porphyrin complexes studied here must originate from their side ligand groups. What remains to be discussed is how the side ligand connects to the observed differences described in the previous paragraphs. Specifically, why does FeOEP-Cl have such a binding interaction with small solvent molecules, while FeTPP-Cl does not? We argue that this difference is due to the geometrical structures of these side ligands as well as their arrangements and locations with respect to the porphyrin molecular plane.

The geometry optimizations of the two porphyrin complexes indicate that both molecules have almost identical center parts, which defines the molecular plane containing the Fe center and all the $\mathrm{N}$ atoms, and also defines the molecular axis along the $\mathrm{Cl}-\mathrm{Fe}$ bond direction perpendicular to the molecular plane (see scheme $\mathrm{S}_{1}$ in the Supporting Information). Because of this similarity, DFT/ROCIS calculations give almost identical theoretical spectra for both porphyrin molecules, shown as blue traces in figure $3 \mathrm{~b}$ and $3 \mathrm{e}$. For the side ligands, eight ethyl groups in FeOEP-Cl complex are all located on one side of the molecular plane with $\mathrm{Cl}^{-}$ion on the other side, giving rise to a total dipole moment of 3.77 Debye according to the DFT geometry optimization. The four phenyl groups (with the phenol molecular plane being almost perpendicular to the porphyrin plane) in FeTPP-Cl, on the other hand, are symmetrically distributed on both sides of the porphyrin molecular plane, resulting in a total 3.09 Debye dipole moment. $\mathrm{FeOEP}-\mathrm{Cl}$ has hence larger electrostatic 
attraction to the polar solvent molecule dichloromethane which has a dipole moment of 1.65 Debye.

When a $\mathrm{CH}_{2} \mathrm{Cl}_{2}$ molecule approaches the Fe center with one of its negatively charged $\mathrm{Cl}$ at the front, an extra $\mathrm{Fe}^{3+}-\mathrm{Cl}^{-}$ion pair may form, which is probable for both porphyrins in solution. Our experimental data combined with the theoretical calculations suggest though that such an ion pair $\left(\mathrm{CH}_{2} \mathrm{Cl}_{2}\right.$ binding to Fe center) is only stabilized in the FeOEP-Cl solution, creating a different ligand field around the Fe center that alters the Fe electronic structure. The stabilization of the binding interaction is probably assisted by the side ligands of FeOEP-Cl. The eight ethyl groups with positive charged $\mathrm{H}$ atoms terminating the side ligands could create an electrostatic field around the porphyrin molecule. ${ }^{45}$ Such a field, when superimposed with the electrical field created by the $\mathrm{Fe}^{3+}$ in the molecular center, causes a local minimum in the energy potential directly below the Fe center along the molecular axis for negative charge. The proposed ligand-assisted binding process for FeOEP-Cl is illustrated in figure 4 . The ligand-induced electrostatic field may help keep the $\mathrm{Cl}$ of the approaching $\mathrm{CH}_{2} \mathrm{Cl}_{2}$ bound to the $\mathrm{Fe}$ center, similar to the "pocket" effect in the case of large protein present in hemoglobin or myoglobin. ${ }^{5}$ The $\mathrm{Fe}^{3+}-\mathrm{Cl}^{-}$ionic attraction, indicated by the red arrows in figure 4, is present in both porphyrin solutions, but may not be strong enough to keep the approaching solvent molecule in the vicinity of the Fe centers if there is no assistance from the ligands. The $\mathrm{H}$ atoms in the phenyl groups of FeTPP-Cl cannot produce a similarly strong electrostatic field to assist the binding process because they are fewer in number and farther in distance to the Fe center. Also, the ethyl groups of FeOEP-Cl are less constraint, and have a larger flexibility to orient their arms in liquid phase, reaching out for nearby $\mathrm{Cl}$ from the solvent molecule to "grab" the dichloromethane molecule. Some of the ethyl arms may also reach to the $\mathrm{Cl}^{-}$ion of the porphyrin itself in solution due to the Coulomb attraction.

It is noteworthy that the left FeOEP-Cl geometric structure in figure 4 (also the top inset in figure 3) is DFT-optimized for the isolated molecule (gas phase). The arrangement of the ethyl groups in the liquid phase may differ when surrounded by numerous solvent molecules. For the extra binding interaction with the solvent molecules, the DFT geometry optimization and the following DFT/ROCIS calculations were only performed on the molecular configuration of the Fe center bound to two $\mathrm{Cl}^{-}$ions (both $\mathrm{Cl}^{-}$are single ions, none being part of $\left.\mathrm{CH}_{2} \mathrm{Cl}_{2}\right)$ in the gas phase for both porphyrins. The arrangement of the eight ethyl groups in the right molecular geometry of figure 4 depicts the binding process in solution proposed here. We believe that such a molecular geometry can assist the binding process, and explains the observed differences in the two porphyrins' experimental spectra. The DFT simulation of the iron porphyrin surrounded by numerous $\mathrm{CH}_{2} \mathrm{Cl}_{2}$ solvent molecules, as occurring in real solutions, exceeds our computation capability. However, it is clear that solvent molecules bind more strongly to the FeOEP-Cl than to the FeTPP-Cl based on our experimental data and DFT/ROCIS calculations. During the proposed binding process (figure 4), the polar and flexible ethyl groups, combined with the larger molecular dipole moment (also induced by the polar side ligands), drive the increased electrostatic interaction between the Fe center of FeOEP-Cl and the small solvent molecule $\mathrm{CH}_{2} \mathrm{Cl}_{2}$ in solution. It should be noted that the chosen solvent molecule $\mathrm{CH}_{2} \mathrm{Cl}_{2}$ is just one example of a test molecule, representing a class of small molecules, such as $\mathrm{O}_{2}, \mathrm{CO}$, etc., existing in real biological system. The finding of the extra binding between the $\mathrm{Fe}$ center and small solvent molecules is not specific to the $\mathrm{Cl}$ of the solvent. Hence, the binding interaction of the Fe center of FeOEP-Cl with other non-chlorinated small molecules is also expected under the ligand assistance. 


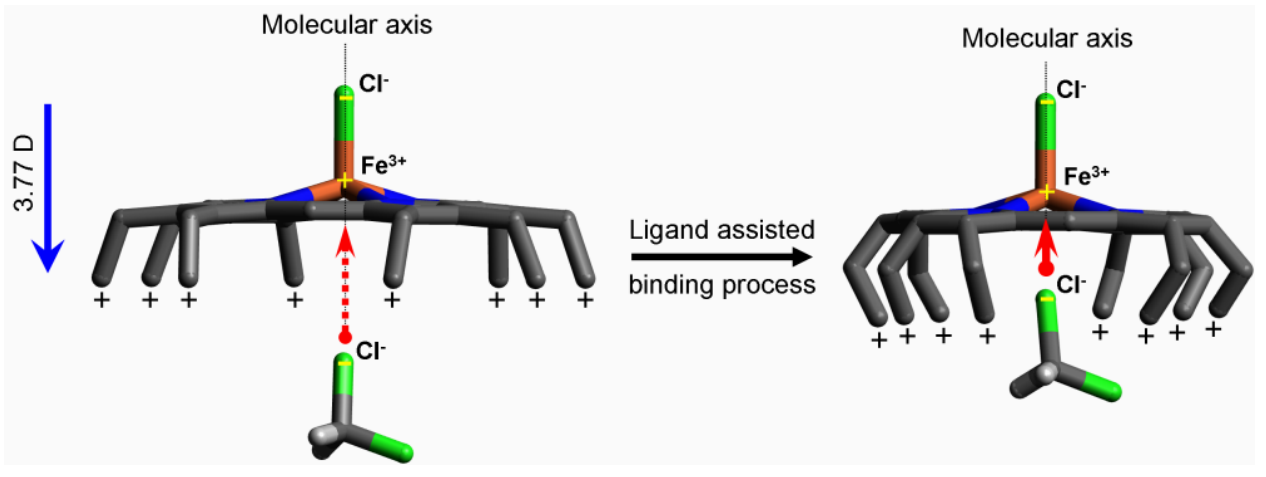

Figure 4. Illustration of the proposed ligand-assisted binding process of $\mathrm{FeOEP}-\mathrm{Cl}$ with $\mathrm{CH}_{2} \mathrm{Cl}_{2}$ solvent molecule. The molecular dipole moment of 3.77 Debye is indicated by a blue arrow on the left for the DFT-optimized original molecular structure (left schematic). The $\mathrm{H}$ atoms in $\mathrm{FeOEP}-\mathrm{Cl}$ are omitted for clearer view except for those terminating the ethyl ligand groups which are represented by "+" signs. Molecular axis is defined along the $\mathrm{Cl}-\mathrm{Fe}$ bond direction and perpendicular to the molecular plane, marked by dashed black lines. The ionic interaction between the $\mathrm{Fe}^{3+}$ center and the $\mathrm{Cl}$ of $\mathrm{CH}_{2} \mathrm{Cl}_{2}$ is indicated by a dashed long red arrow (left) when the solvent molecule is far and the binding is weak, and a solid short red arrow when the solvent molecule is close to the $\mathrm{Fe}^{3+}$ center and the binding becomes stronger (right).

\section{Conclusion}

Our soft X-ray absorption and emission spectroscopy measurements, combined with DFT/ROCIS calculations, strongly indicate that the Fe center of $\mathrm{FeOEP}-\mathrm{Cl}$ porphyrin has a stronger binding interaction with the solvent molecule $\mathrm{CH}_{2} \mathrm{Cl}_{2}$ in solution when compared to FeTPP-Cl. This difference is mainly attributed to the different extent of the ligand assistance to the binding process. Our finding will help understand, on the microscopic level, how $\mathrm{O}_{2}$ or other small molecules bind to iron porphyrin in biologically relevant systems. This work also motivates future researches on the artificial tuning of the porphyrin functionality by modifying the side ligands.

\section{SUPPORTING INFORMATION}

DFT calculations of molecular geometries, orbitals, and simulated XAS spectra. This material is available free of charge via the Internet at http://pubs.acs.org.

\section{AUTHOR INFORMATION}

\section{Corresponding Author}

* emad.aziz@helmholtz-berlin.de

* jie.xiao@helmholtz-berlin.de

\section{Author Contributions}

The manuscript was written through contributions of all authors. All authors have given approval to the final version of the manuscript.

\section{ACKNOWLEGEMENT}

This work was supported by the European Research Council grant No. 279344. Part of this work was financially supported by the Helmholtz-Gemeinschaft via the young investigator fund VH-NG-635. E.F.A and K.A. would like to acknowledge the financial support of the Einstein Foundation Berlin for the postdoctoral scholarship in the Aziz team.

\section{Reference}

(1) Collman, J. P.; Brauman, J. I.; Iverson, B. L.; Sessler, J. L.; Morris, R. M.; Gibson, Q. H. J. Am. Chem. Soc. 1983, 105, 3052.

(2) Pauling, L.; Coryell, C. D. Proc. Natl. Acad. Sci. 1936, 22, 210.

(3) Shaanan, B. J. Mol. Biol. 1983, 171, 31.

(4) Shikama, K. Chem. Rev. 1998, 98, 1357.

(5) Springer, B. A.; Sligar, S. G.; Olson, J. S.; Phillips, G. N. J. Chem. Rev. 1994, 94, 699.

(6) Weiss, J. J. Nature 1964, 202, 83.

(7) Wilson, S. A.; Kroll, T.; Decreau, R. A.; Hocking, R. K.; Lundberg, M.; Hedman, B.; Hodgson, K. O.; Solomon, E. I. J. Am. Chem. Soc. 2013, 135, 1124.

(8) Chen, H.; Ikeda-Saito, M.; Shaik, S. J. Am. Chem. Soc. 2008, 130, 14778.

(9) Hocking, R. K.; Wasinger, E. C.; Yan, Y.-L.; deGroot, F. M. F.; Walker, F. A.; Hodgson, K. O.; Hedman, B.; Solomon, E. I. J. Am. Chem. Soc. 2007, 129, 113.

(10) Aziz, E. F.; Ottosson, N.; Bonhommeau, S.; Bergmann, N.; Eberhardt, W.; Chergui, M. Phys. Rev. Lett. 2009, 102, 068103.

(11) Harada, Y.; Taguchi, M.; Miyajima, Y.; Tokushima, T.; Horikawa, Y.; Chainani, A.; Shiro, Y.; Senba, Y.; Ohashi, H.; Fukuyama, H.; Shin, S. J. Phys. Soc. Jpn. 2009, 78, 
044802.

(12) Lange, K. M.; Golnak, R.; Bonhommeau, S.; Aziz, E. F. Chem. Commun. 2013, 49, 4163.

(13) Collman, J. P.; Brauman, J. I.; Halbert, T. R.; Suslick, K. S. Proc. Natl. Acad. Sci. 1976, 73, 3333.

(14) Pauling, L. Nature 1964, 203, 182.

(15) Phillips, S. E. V.; Schoenborn, B. P. Nature 1981, 292, 81

(16) Traylor, T. G.; Koga, N.; Deardurff, L. A.; Swepston, P. N.; Ibers, J. A. J. Am. Chem. Soc. 1984, 106, 5132.

(17) Traylor, T. G.; Tsuchiya, S.; Campbell, D.; Mitchell, M.; Stynes, D.; Koga, N. J. Am. Chem. Soc. 1985, 107, 604.

(18) Chou, K.-C. Trends Biochem. Sci. 1989, 14, 212.

(19) Suljoti, E.; Garcia-Diez, R.; Bokarev, S. I.; Lange, K. M.; Schoch, R.; Dierker, B.; Dantz, M.; Yamamoto, K.; Engel, N.; Atak, K.; Kühn, O.; Bauer, M.; Rubensson, J.-E.; Aziz, E. F. Angew. Chem. Int. Ed. 2013, 52, 9841.

(20) Atak, K.; Bokarev, S. I.; Gotz, M.; Golnak, R.; Lange, K. M.; Engel, N.; Dantz, M.; Suljoti, E.; Kühn, O.; Aziz, E. F. J. Phys. Chem. B 2013, 117, 12613

(21) Bokarev, S. I.; Dantz, M.; Suljoti, E.; Kühn, O.; Aziz, E. F. Phys. Rev. Lett. 2013, 111, 083002.

(22) Lange, K. M.; Kothe, A.; Aziz, E. F. Phys. Chem. Chem. Phys. 2012, 14, 5331.

(23) Lange, K. M.; Aziz, E. F. Chem. Soc. Rev. 2013, 42, 6840 .

(24) Aziz, E. F. J. Phys. Chem. Lett. 2011, 2, 320.

(25) De Groot, F. Chem. Rev. 2001, 101, 1779.

(26) Lange, K. M.; Könnecke, R.; Ghadimi, S.; Golnak, R.; Soldatov, M. A.; Hodeck, K. F.; Soldatov, A.; Aziz, E. F. Chem. Phys. 2010, 377, 1.

(27) Soldatov, M. A.; Lange, K. M.; Gotz, M. D.; Engel, N.; Golnak, R.; Kothe, A.; Aziz, E. F. Chem. Phys. Lett. 2012, 546, 164.

(28) Gotz, M. D.; Soldatov, M. A.; Lange, K. M.; Engel, N.; Golnak, R.; Könnecke, R.; Atak, K.; Eberhardt, W.; Aziz, E. F. J. Phys. Chem. Lett. 2012, 3, 1619.

(29) Neese, F. Wiley Interdiscip. Rev. Comput. Mol. Sci. 2012, 2, 73.

(30) Becke, A. D. Phys. Rev. A 1988, 38, 3098.

(31) Becke, A. D. J. Chem. Phys. 1993, 98, 5648.

(32) Weigend, F.; Ahlrichs, R. Phys. Chem. Chem. Phys. 2005, 7, 3297.

(33) Roemelt, M.; Maganas, D.; DeBeer, S.; Neese, F. J. Chem. Phys. 2013, 138, 204101.

(34) Roemelt, M.; Neese, F. J. Phys. Chem. A 2013, 117, 3069.

(35) Baerends, E. J.; Ellis, D. E.; Ros, P. Chem. Phys. 1973, 2, 41

(36) Dunlap, B. I.; Connolly, J. W. D.; Sabin, J. R. J. Chem. Phys. 2008, 71, 3396.

(37) Vahtras, O.; Almlöf, J.; Feyereisen, M. W. Chem. Phys. Lett. 1993, 213, 514.

(38) Eichkorn, K.; Treutler, O.; Öhm, H.; Häser, M.; Ahlrichs, R. Chem. Phys. Lett. 1995, 240, 283.
(39) Eichkorn, K.; Weigend, F.; Treutler, O.; Ahlrichs, R. Theor. Chem. Acc. 1997, 97, 119.

(40) Weigend, F. Phys. Chem. Chem. Phys. 2006, 8, 1057.

(41) Charkin, O. P.; Klimenko, N. M.; Charkin, D. O.; Chang, H.-C.; Lin, S.-H. J. Phys. Chem. A 2007, 111, 9207.

(42) Koenig, D. F. Acta Crystallogr. 1965, 18, 663.

(43) Kotani, M. Ann. N. Y. Acad. Sci. 1969, 158, 20.

(44) Suchkova, S. A.; Soldatov, A.; Dziedzik-Kocurek, K.; Stillman, M. J. J. Phys. Conf. Ser. 2009, 190, 012211.

(45) Zypman, F. R. Am. J. Phys. 2006, 74, 295. 
SYNOPSIS TOC

Insert Table of Contents artwork here

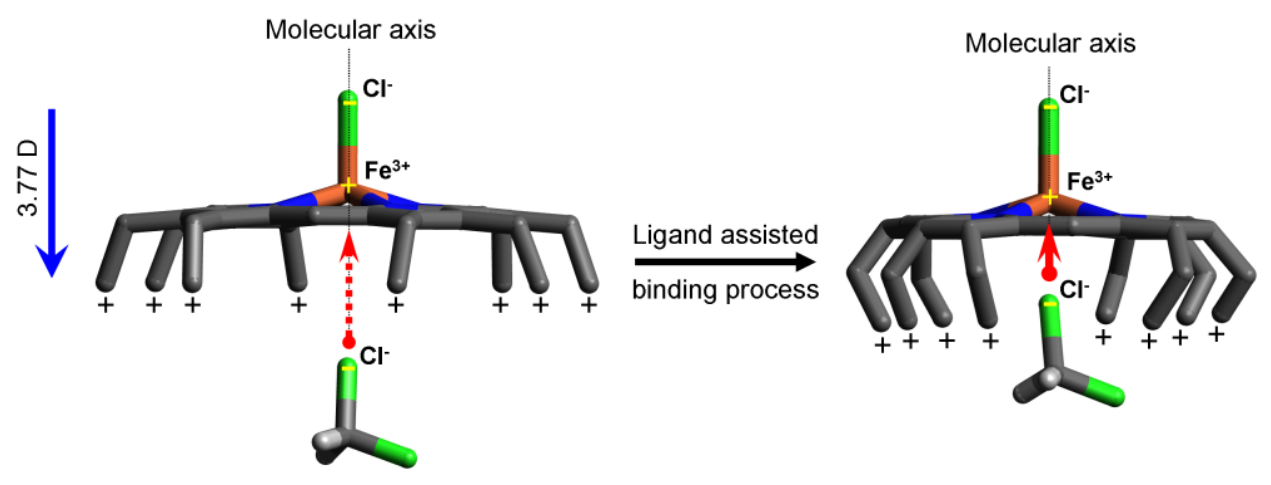

